\title{
Editorial: Molecular and Cellular Physiology of Gametes in Domestic and Wild Animal Models
}

\author{
Silvina Perez-Martinez ${ }^{1}$, Ana Josefa Soler ${ }^{2}$ and Andreina Cesari ${ }^{3 *}$ \\ ${ }^{1}$ Centro de Estudios Farmacológicos y Botánicos (CEFYBO UBAVCONICET), Buenos Aires, Argentina, ${ }^{2}$ IREC (UCLM-CSIC- \\ JCCM), ETSIAM Campus Universitario, Albacete, Spain, ${ }^{3}$ Instituto de Investigaciones Biologicas (IIB CONICET/UNMdP), Mar del \\ Plata, Argentina
}

Keywords: sperm, oocyte, seminal plasma, embryo, development, wild animals, domestic animals

Editorial on the Research Topic

Molecular and Cellular Physiology of Gametes in Domestic and Wild Animal Models

Gamete physiology contributes not only to the zygote formation but also to early embryo development. In mammals, most of the knowledge has been acquired from research on the murine and human models. This topic fills the information gap related to the effect of male and female microenvironments over sperm physiology from different species and offers critical and new insights into the molecular mechanism of gametes' maturation to acquire fertilizing competence in wild and domestic animals.

It includes 16 original research articles divided into three Research Topic: 1) oocyte maturation; 2) sperm fertilizing ability in response to female reproductive environment, and 3) molecular evaluation of seminal plasma related to sperm quality.

1) Oogenesis has been studied in mammalian and non-mammalian organisms, however to date, the mouse has been the most studied model. Knowledge of how conserved or distinctive are both nuclear and epigenetic molecular mechanisms of oocyte maturation and development in farm and wild animals would help to delineate complex molecular mechanisms and identify major genes and proteins involved.

In this direction, nuclear proteins such as chromatin and ribosomal binding proteins have been identified as conserved molecular actors. Here, Chavez et al. identify nuclear BRD2 and NPM1 as novel factors that contribute to oocyte meiotic competence in the domestic cat. By transfecting complex-oocyte-cumulus with specific antibodies against these proteins they showed that BRD2 and NPM11 inhibited in vitro fertilization and oocytes were arrested at the germinal vesicle (GV) stage. Other nuclear proteins are known to interact with the kinetochore during the spindle formation controlling chromosome segregation during meiosis. Unlike rodents, mares have prolonged reproductive scenes and it is shown that three spindle related mRNAs are downregulated during aging: Mps1, Spc25 and AurkC (Rizzo et al.). Moreover, after pharmacological inhibition of MPS1 kinase, MII oocytes from aged mares showed a higher incidence of spindle abnormalities while AURKC inhibition severely impaired microtubule organization and spindle formation in all oocytes, irrespective of mare age. On the other hand, non-nuclear proteins have been also found to be implicated in spindle organization during oocyte maturation. In sheep, Han et al. showed that CLTC is localized in the oocytes spindle after GV break-down. Knockdown of the membrane protein CLTC by morpholino injection into GV oocytes disrupted spindle assembly and chromosome alignment, accompanied by impaired first polar body emission and altered early embryo development.

Regarding extracellular factors, the dialog between the oocyte and surrounding granulosa/ cumulus cells operates through cytoplasmic projections-gap-junctions and through soluble molecules with specific receptors. Idrees et al. explores a novel role for a SHP2-ERa complex in the nucleus of bovine cumulus cells. The SHP2/ERa complex is highly expressed in the cumulus cells 
of immature oocytes (GV) and reduced in the cumulus cells of mature oocytes (MII). They showed that the formation of a SHP2/ERa complex promotes NPPC and NPR2 transcription (genes related to meiotic arrest) and that meiotic resumption controlled by FSH or growth factors, restricts SHP2-ERa interaction by promoting its extrusion from the nucleus.

In targeted species for the productive sector, successful in vitro oocyte maturation is required for in vitro embryo production. These biotechnologies produce cellular stress and one of the main mechanisms is associated with the endoplasmic reticulum (ER). Pioltine et al. provide insight into the role of TUDCA (tauroursodeoxycholic acid) in relieving ER stress by decreasing reactive oxygen species (ROS) production in bovine oocytes. This bile acid acts as a potent chemical chaperone and increases the abundance of transcripts related to antioxidant activity in bovine oocytes (CAT, GPX1, and HMOX1) and embryos (GPX1 and PRDX3). In contrast to other mammals, oocytes of dogs and foxes are ovulated at the GV stage and undergo meiotic maturation within the oviduct (Viveiros and De la Fuente, 2019). For this reason, in vitro maturation to MII is not successful and most oocytes degenerate. The work by Duque Rodriguez et al. addresses the supplementation of media with BSA and fetal bovine serum in the presence of insulin-transferrinselenium. Insulin promotes the absorption of nutrients, while transferrin and selenium are antioxidants. They found a condition that increased the proportion of oocytes at the MII stage, with a higher integrity of the cytoplasmic membrane and lower ROS levels.

These last three works underline the in vivo and in vitro effects of environmental factors on oocyte and embryo quality.

2) Sperm exposure to the female reproductive tract leads them to constantly changing environments, from which they receive different chemical signals (Plant and Zeleznik, 2014). This set of inputs drives a series of signaling events that are essential for sperm capacitation, a process that confers spermatozoa the ability to fertilize an oocyte (Chang, 1951; Austin, 1952). After entering the female tract, spermatozoa find higher bicarbonate and calcium levels, whose influx activates cAMP-PKA pathway and increases tyrosine phosphorylation downstream, thus hyperactivating sperm motility. This ion flow through the sperm plasma membrane triggers lipid reorganization, cholesterol efflux, and consequently, membrane hyperpolarization that increases its fusogenicity. Exposure to the female reproductive tract involves a major challenge to sperm ability to adapt to drastic osmolality variations. In this sense, the results presented from Delgado-Bermudez et al. showed the essential role of aquaporins in osmoregulation in order to maintain sperm motility and lipid architecture during sperm capacitation. They also found that aquaporins might have a relevant role in sperm signaling pathways involved in acrosome reaction in pigs.

On the other hand, ROS are essential for processes like capacitation, hyperactivation, acrosome reaction, and spermoocyte fusion, although an excess can be detrimental to these processes. In ovine, reproduction has a marked seasonality controlled by melatonin secretion from the pineal gland. Miguel-Jimenez et al. described how melatonin, also presented in the reproductive fluids, directly affects ram sperm functionality and exerts an antioxidant role, decreasing oxidative stress, by modulating NOX5 activity during capacitation.

About fifty percent of male subfertility diagnosis is idiopathic and is usually associated with genetic abnormalities or protein dysfunction, which are not detectable through the conventional spermiogram. Recent studies have evidenced the essential role of antioxidant enzymes, such as GSTP, in sperm protection against oxidative stress and preservation of sperm function and fertilizing ability. Llavanera et al. found that the dissociation of the GSTP1-JNK heterocomplex results in the activation of JNK and significantly declines sperm viability, motility, mitochondrial activity and plasma membrane stability. This study evaluated the function of this heterocomplex in pig sperm physiology.

Many sperm-specific proteins and their underlying molecular activation mechanisms involved in sperm physiology of most vertebrates still remain unknown. As transcription and translation are highly limited in sperm, proteins are key molecules defining their function, making proteomic approaches one of the most adequate methods to investigate sperm fertilizing ability. In particular, the molecular basis of male fertility in chickens is still unexplored. Here, Carvalho et al. identified 57 proteins related to fertility in meat-type roosters. These proteins were involved in various molecular pathways including flagellum integrity and movement, mitochondrial function, sperm maturation, and storage in the female tract as well as oocyte-sperm interaction. This relevant study about avian reproduction may impact future work on bird fertility and conservation of species.

Spermatozoa are highly differentiated cells that present their chromatin mainly condensed in protamines to protect genetic information from damaging agents, facilitating the delivery of an intact genetic material to the future embryo. This topic includes a study that describes species-specific differences in the decondensation capacity of sperm DNA, and especially in DNA condensation type around protamine 1 and protamine 2 or only protamine 1 (Ribas-Maynous et al.).

Here are also shown results related to the expression and the regulation of circRNAs in sheep testis. Li et al. elucidated for the first time, circRNA dynamic expression patterns across three reproductive stages in postnatal sheep testes, They also described circRNA-associated miRNA-gene network implicated in testicular function such as spermatogenesis, germ cell development, blood-testis barrier and cell cycle/meiosis. These gene tools could be helpful for providing new insights into the molecular mechanism of ovine testis function.

3) The extracellular environment determines gamete physiology. In the case of the male gamete there are two different natural milieus contributing to intracellular changes: the seminal plasma (SP) and the female fluids. SP interchanges molecules with spermatozoa that promote their acquisition of fertilizing ability. In addition, proteins from reproductive fluids interact with the sperm proteome and differ among species. This topic includes two works related to enzymes and metabolites from pig SP. Llavanera et al.used a RMN analysis finding 23 metabolites categorized as: amino acids, saccharides, salts, 
alcohols/other organic compounds. To look for potential biomarkers, they search for associations between those molecules and breed, fresh sperm quality/functionality parameters and resilience to liquid storage. They found that only fumarate followed a breed-dependent behavior, while they could associate metabolite patterns from all the categories with specific sperm parameters. Delgado-Bermúdez et al. characterize AKR1B1 from pig SP, a NADPH-dependent enzyme involved in fructose production. They found higher concentrations of AKR1B1 in the post sperm-rich fraction of SP and it was detected in most boar male reproductive tissues. This enzyme was not related to sperm quality/functionality or to the sperm ability to withstand liquid storage.

Little information concerning reproductive features is available in the case of aquatic animals like cetaceans. Furthermore, the importance of studying this animal model is under enormous anthropogenic pressure as a result of commercial overfishing, incidental captures in fisheries and habitat degradation. Indeed, many cetacean species are threatened or in danger of extinction. The work developed by Fuentes-Albero et al. is the first characterization of the proteome in cetacean sperm and SP, which could be an important contribution to future research into new biomarkers, the analysis of conservation capacity, or possible additional applications in the field of assisted reproductive technologies.

Post-thaw sperm quality is quite different among animal species as a result of their different sensitivity to freezing and thawing. The low survival rate of cryopreserved sperm is associated with structural and functional alterations. In this sense, the low sterol/phospholipid ratio of ram sperm membranes has been identified as one of the most significant factors determining their low tolerance to cold

\section{REFERENCES}

Austin, C. R. (1952). The 'Capacitation' of the Mammalian Sperm. Nature 170 (4321), 326. doi:10.1038/170326a0

Carro, M. D. L. M., Peñalva, D. A., Antollini, S. S., Hozbor, F. A., and Buschiazzo, J. (2020). Cholesterol and Desmosterol Incorporation into Ram Sperm Membrane before Cryopreservation: Effects on Membrane Biophysical Properties and Sperm Quality. Biochim. Biophys. Acta (Bba) Biomembranes 1862 (9), 183357. doi:10.1016/j.bbamem.2020.183357

Chang, M. C. (1951). Fertilizing Capacity of Spermatozoa Deposited into the Fallopian Tubes. Nature 168, 697-698. doi:10.1038/168697b0

Plant, T., and Zeleznik, A. (2014). "Knobil and Neill's Physiology of Reproduction Physiology of Reproduction," in Section I: Section I. Gametes, Fertilization and Embryogenesis. Editors T. Plant and A. Zeleznik. 4th Edition. doi:10.1016/ C2011-1-07288-0

Viveiros, M. M., and De La Fuente, R. (2019). "Regulation of Mammalian Oocyte Maturation," in Chapter 11 - Regulation of Mammalian Oocyte Maturation temperatures. Recently, Carro et al. (2020) found a positive effect on membrane biophysical properties in ram sperm adding cholesterol and desmosterol into sperm extenders prior to cryopreservation. In this topic, these authors (Carro et al.) studied the role of cholesterol and desmosterol in ram sperm functionality and fertility, proposing the benefit of the incorporation of desmosterol as a strategy to improve fertility outcome.

The works presented in this topic provide meaningful information on studying molecular and cellular processes that give fertilizing competence in spermatozoa and oocytes of nonmurine or non-human animals. These findings also contribute to the knowledge of new insight into molecular mechanisms to develop biotechnological tools for improving domestic animal production and for the conservation of biodiversity of wild species.

\section{AUTHOR CONTRIBUTIONS}

SP-M, AC, and AV drafted and further revised the manuscript. All authors listed have read this editorial and approved it for publication.

\section{ACKNOWLEDGMENTS}

As Research Topic editors we would like to express our sincere thanks to all authors and referees for their valuable contributions, which made it possible for us to compile this interesting Research Topic on Molecular and Cellular Physiology of Gametes in Domestic and Wild Animal Models.

(165-180), the Ovary. Editors P. C. K. Leung and E. Y. Adashi. Third Edition (Academic Press), 165-180. doi:10.1016/B978-0-12-813209-8.00011-X

Conflict of Interest: The authors declare that the research was conducted in the absence of any commercial or financial relationships that could be construed as a potential conflict of interest.

Publisher's Note: All claims expressed in this article are solely those of the authors and do not necessarily represent those of their affiliated organizations, or those of the publisher, the editors and the reviewers. Any product that may be evaluated in this article, or claim that may be made by its manufacturer, is not guaranteed or endorsed by the publisher.

Copyright (c) 2021 Perez-Martinez, Soler and Cesari. This is an open-access article distributed under the terms of the Creative Commons Attribution License (CC BY). The use, distribution or reproduction in other forums is permitted, provided the original author(s) and the copyright owner(s) are credited and that the original publication in this journal is cited, in accordance with accepted academic practice. No use, distribution or reproduction is permitted which does not comply with these terms. 